\title{
sols frottants mobiles et mobilisables sous l'action de la houle et des courants en géotechnique marine
}

A. GROVEL

Professeur*

P. THOMAS

Assistant*

\section{INTRODUCTION}

\subsection{Position du problème}

L'étude géotechnique des sols marins de surface présente dès l'abord deux difficultés spécifiques:

a. le sol marin est un sédiment, un dépôt, en présence ou non des forces qui l'ont mis en place,

b. le mode de prélèvement est toujours plus perturbateur qu'à terre et la possibilité de mélange des couches est toujours possible.

Schématiquement, si l'on admet qu'un sol d'été est formé par la superposition de deux couches:

- l'une en équilibre statistique, le mouvement des grains vers l'aval étant compensé par l'apport d'amont, sous les forces hydrauliques,

- l'autre stable.

L'apparition des forces maximales d'hiver va balayer le sol superficiel, qui reparaîtra à l'été suivant.

(*) 1.U.T. de Saint-Nazaire

E.N.S.M. de Nantes - 1, rue de la Noë - 44300 Nantes.

\begin{tabular}{lll}
$\frac{\text { ÉTÉ 1 }}{\text { Sol 1 }}$ & HIVER & $\frac{\text { ÉTÉ 2 }}{\text { Sol 1 }}$ \\
\hline Sol 2 & Sol 2 & Sol 2
\end{tabular}

Il paraissait donc important de mettre au point une méthode permettant de préciser:

- d'une part à quel sol de surface on a affaire (stabilité),

- d'autre part comment se répartissent en profondeur les sols d'occurrence de mobilité variables.

Le paramètre essentiel est donc la profondeur de mobilité dont l'influence sur les comportements de fondations à l'affouillement fait pressentir l'intérêt.

$\mathrm{Ce}$ paramètre a une signification en géotechnique différente de la notion de "couche mobile" de l'hydraulique: il intègre dans l'espace et dans le temps les phénomènes de transport, de début de remise en mouvement, de dépôt, alors que la «couche mobile» est la partie supérieure du sol qui instantanément sous une force hydraulique quelconque est susceptible de mouvement; l'épaisseur de la couche mobile est de l'ordre de quelques diamētres de grains et ne saurait 
donc expliquer les phénomènes d'ensouillage ou d'affouillement.

Le schéma suivant illustre cette distinction.

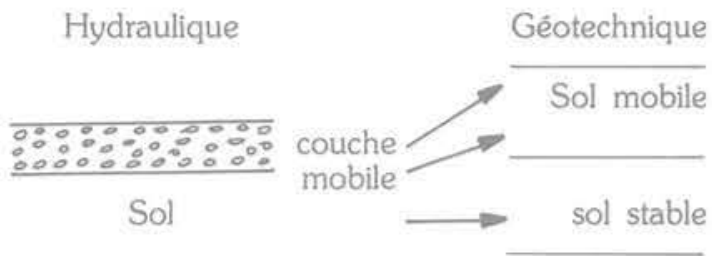

\subsection{Expression de l'hypothèse de travail de départ}

Devant les difficultés éprouvées par la plupart des spécialistes de fonds marins à appliquer en nature les résultats des laboratoires hydrauliques, il fut donc décidé:

- de s'en tenir uniquement aux sols frottants,

- de reprendre le problème au début.

\subsubsection{Les lois de début d'entraînement des sédi- ments}

De nombreuses mesures de laboratoire ont été effectuées sur des sphères de matériaux divers et BONNEFILLE et PERNECKER en ont publié en 1966 une loi de synthèse exprimée par la relation générale

$$
r^{2}=\frac{2}{3} f D^{3}
$$

- oû $f$ est le coefficient de frottement unitaire d'un grain

$f=\operatorname{tg}_{\mu}^{\varphi}$
- R. le nombre de Reynolds étoilé $\frac{\mu_{*} \mathrm{D}}{v}$

u. vitesse de cisaillement $\sqrt{\frac{\tau_{0}}{\rho}}$ racine du rapport de

la contrainte de cisaillement au fond sur la masse spécifique de l'eau.

$\mathrm{D}$ dimension diamètre du grain.

$v$ viscosité cinématique de l'eau.

- D. diamètre sédimentologique $=\left(\frac{\rho^{\prime} g}{v^{2}}\right)^{1 / 3}$

où $\rho^{\prime}=\frac{\rho_{s}-\rho}{\rho}$

Cette loi (de début de mise en mouvement) est obtenue par l'égalité des forces de frottement et de traction en utilisant les formules de STRICKLER valables pour un écoulement en canal et reliant ainsi vitesse moyenne, profondeur d'écoulement et dimension du grain au coefficient de CHEZY.

La première difficulté rencontrée dans son application éventuelle à un phénomène naturel, concerne la signification de D présenté comme une valeur moyenne.
Il s'agit ici du diamètre moyen d'une population statistique de billes artificielles dont l'écart-type est très réduit.

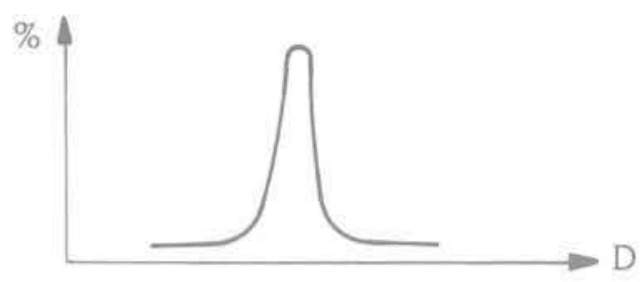

En nature la définition du diamètre moyen concerne une population aux dimensions plus étalées, quand ce n'est pas le mélange de plusieurs populations.

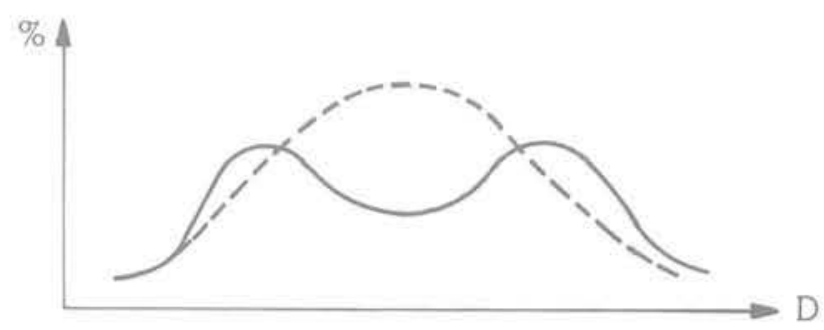

Les lois de synthèse sont donc peu appliquées principalement du fait que le paramètre adiamètre moyen» est peu adapté.

1.2.2. Recherche d'un paramètre dimensionnel représentatif d'une population statistique de sols frottants

L'hypothèse de travail dont nous sommes partis peut se résumer ainsi :

$\mathrm{Si}$, sur le plan sédimentologique, nous avons la preuve, par des tests ou des observations, qu'un sable est mis en mouvement par un courant ou une houle,

- étant donné qu'il existe une relation bi-univoque entre la vitesse du courant ou la vitesse orbitale des houles et la dimension des particules.

- étant donné d'autre part que la répartition statistique de ces vitesses est toujours gaussienne (loi normale de rêpartition), la répartition statistique des dimensions des particules devrait être elle aussi normale.

La première difficulté provient du fait que la population de grains de sable n'est pas comptée mais répartie en pourcentages de poids soit par tamisage soit par élutriation.

La distribution des pourcentages de poids en fonction des dimensions suit donc alors tout naturellement la loi log-normale

$$
\begin{aligned}
& -A=-\frac{1}{2}\left(\frac{L . D-m}{\sigma}\right)^{2} \begin{array}{r}
m \text { moyenne } \\
\sigma \text { écart-type }
\end{array} \\
& -f(\log D)=\frac{1}{\sigma \sqrt{2 \pi}} e^{A}
\end{aligned}
$$


Cette distribution couramment utilisée présente deux particularités dont nous avons décidé de tirer parti:

a. la courbe cumulative des résultats d'un simple tamisage représentée en abscisse logarithmique de dimensions et en ordonnées de probabilité permet d'utiliser le test graphique de HENRY et de déterminer ainsi si tout ou partie de la courbe suit une loi log-normale.

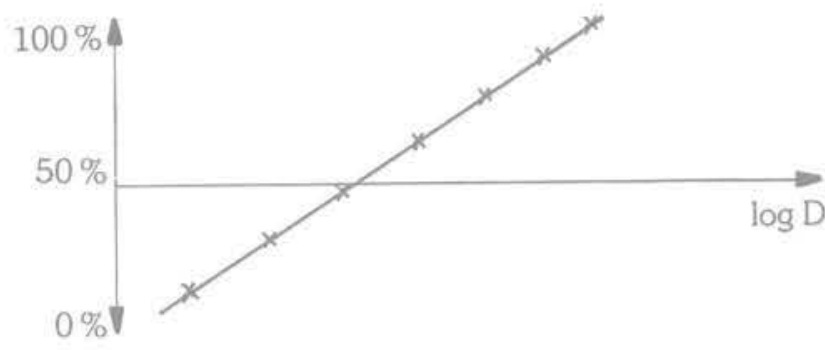

b. de même que la somme de deux répartitions normales est elle-même normale, c'est le produit de deux répartitions log-normales qui est log-normal.

Le mélange de deux populations de sables à répartition log-normale ne peut donc pas suivre une répartition log-normale et dans une courbe granulométrique cumulative les droites de Henry ne représenteront que des domaines où la répartition des dimensions a été régie par une force physique de répartition normale, unique ou prédominante.

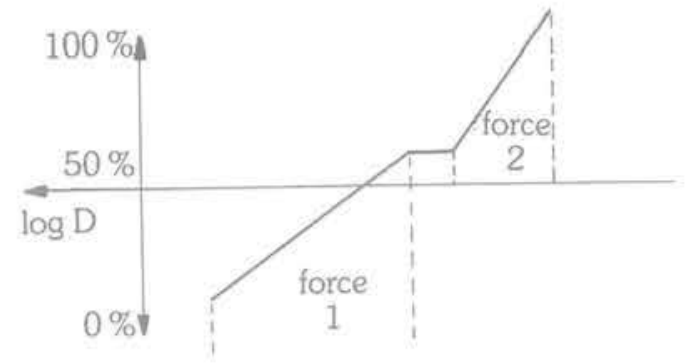

Or, les zones étudiées ont montré des diagrammes typiques de deux populations,
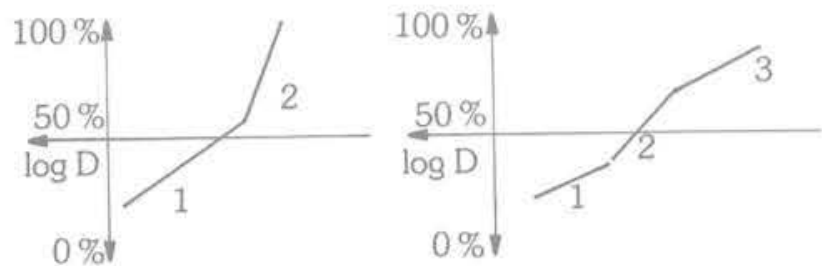

rarement de 3 populations. Pour savoir si le diagramme "a* pouvait être considéré ou non comme un sol d'été surmontant un sol d'hiver, et qu'on avait alors affaire à ces deux sols mélangés par l'opération de prélèvement par benne, une opération test fut entreprise dans une zone témoin.

\section{TEST DANS UNE ZONE A COURANTOLOGIE CONNUE}

\subsection{Situation générale}

La zone considérée est une zone sableuse à l'abri de l'île de Groix et à la sortie de la rade de Lorient. Un houlographe mouillé sur le site (au point I) a enregistré sur 330 jours de mesure, 247 jours de mer zéro.

Il est cependant évident que certaines tempêtes arrivent à influencer cette zone et les 15 jours annuels où la houle dépasse 1 mètre de creux, peuvent voir apparaître des houles maximales suivantes:

$\begin{array}{ccc}\text { Direction } & \text { Période } & \mathrm{H} \max . \\ \text { W-SW } & 7 \mathrm{~s} & 2,70 \mathrm{~m} \\ \text { SW } & 10-14 \mathrm{~s} & 2,30 \mathrm{~m} \\ \text { S-SE } & 5 \mathrm{~s} & 2,65 \mathrm{~m}\end{array}$

Il est difficile de trouver une zone marine atlantique plus à l'abri des houles.

Les courants ont donc été mesurés par 17 mouillages (à mi-profondeur) de courantographes automatiques de 15 à 20 jours chacun, donnant des résultats assez proches de la vitesse moyenne de la tranche d'eau $\hat{\mathrm{V}}$.

Le courant a été analysé entre ses différentes composantes:

- courant de marée

- courant de dérive (dâ au vent)

- courant de vidange de l'estuaire (dû aux débits des fleuves).

Une prédiction pragmatique en a été tirée, mais surtout une série de lâchers de flotteurs dérivants a permis de tracer une carte des différents chenaux (flot et jusant) suivant les situations météorologiques.

A première vue, il semble que la variation de position des chenaux de vitesses, doive donner une valeur moyenne en tout point mais la fréquence prédominante des vents d'ouest a cependant tendance à conserver un chenal de flot le long de la côte au Nord, et un chenal de jusant plutôt au Sud.

\subsection{Exploitation des données dimension- nelles}

Les sables ont été prélevés par dragages l'été au cône Berthois et leurs dimensions déterminées par tamisage ou élutriation au tube d'Emery.

La rupture de la courbe log-gaussienne a été prise comme dimension maximale du grain pouvant être remis en mouvement par les courants d'été (dérive d'Ouest faible et coefficients maximaux 95).

L'hypothèse de travail était donc que la houle d'été inexistante ou très faible ne pouvait remuer qu'une 
population très fine (Max 2) la population la plus grossière étant représentative du courant (Max 1).

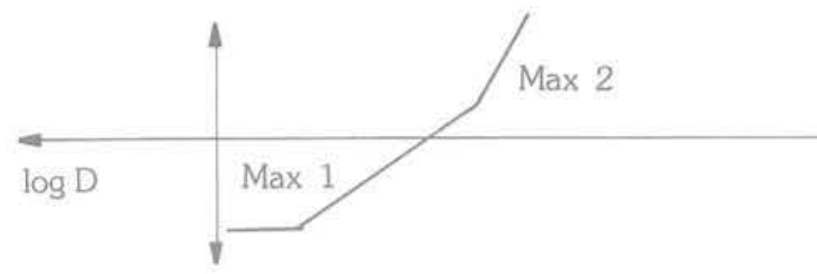

On n'a donc en général que des $D$ compris entre 1 et $2 \mathrm{~mm}$

En appliquant la loi de BONNEFILLE avec $\mathrm{f}=1$, on peut reconstituer une carte de courants fictifs qui permet de visualiser trois faits:

a. des grandes valeurs au Nord sont en concordance avec le chenal de flot correspondant à la dérive $W$.

b. le chenal de jusant du Sud est moins net.

c. une zone au SW oũ l'interprétation des phénomènes naturels est plus difficile.

C'est une zone morphologiquement complexe (ancienne barre des gêographes) et les maxi 1 et 2 ont peut-être ici leurs rôles inversés (zone plus ouverte à la houle).

\subsection{Remarques}

Il était difficile d'aller plus loin surtout avec une analyse de courants. Cependant l'hypothèse de départ n'était pas infirmée. Il semblait alors plus intéressant de travailler sur une zone soumise à la houle où la répartition dans l'espace des vitesses orbitales sur le fond du fait de la répartition était considérablement plus variée.

3. INFLUENCE DE LA HOULE SUR LA TENUE D'UN SOL MARIN PULVÉRULENT

\subsection{Présentation du site choisi}

Pour mettre en évidence l'influence de la houle sur la tenue d'un sol marin pulvérulent, nous avons choisi un site d'un grand intérêt scientifique et économique: l'estuaire de la Loire au large d'une ligne Saint-MarcSaint-Brévin.

La connaissance du comportement des sols marins y est très précieuse en raison de son influence sur les importants travaux de dragage du chenal d'accès mis en œuvre par le Port Autonome de Nantes SaintNazaire.

L'estuaire externe de la Loire peut être subdivisé en 2 zones marines fortement différenciées: a. Le littoral nazairien est soumis à l'influence des courants fluviaux dont les caractéristiques varient beaucoup selon les saisons. Leur importance est, en général, telle que le profil des marées y est fortement dissymétrique: la période de jusant étant beaucoup plus longue que celle de flot. La Loire charrie, dans cette région, protégée naturellement de la houle, de la vase et quelques limons d'origine terrigène. Le sol marin y est de nature cohésive.

b. La zone située au sud d'une ligne Saint-Marc-SaintBrévin est soumise aux contraintes engendrées par la houle dont la propagation ne subit aucune atténuation au large puisqu'il n'y a pas d'ile jouant le rôle d'amortisseur naturel tel Groix devant Lorient. L'influence des courants fluviaux sur le remaniement des fonds $y$ est très faible: on n'y trouve que très exceptionnellement de rares sédiments d'origine terrigène, les sols sont généralement pulvérulents, avec de fréquentes populations coquillères importantes. C'est donc à cette dernière zone que nous nous sommes tout particulièrement intéressés.

\subsection{Interprétation de l'influence des houles sur les sols marins}

\subsubsection{Interprétation qualitative}

STOKES a décrit les principales caractéristiques de la houle telle qu'on peut la percevoir en première approximation (ce qui est tout à fait suffisant ici, compte tenu du caractère appliqué de la présente étude): la houle est sinusoïdale, sa longueur d'onde varie avec la profondeur, les particules décrivent des trajectoires elliptiques fermées dont les orbites diminuent rapidement quand on s'approche du fond où elles sont animées d'un mouvement rectiligne alternatif. L'oscillation induite sur les sédiments pulvérulents en place ne peut apparaître que si la contrainte tractrice, proportionnelle à l'amplitude de la houle et inversement proportionnelle à la profondeur, est supérieure à la contrainte critique de cisaillement, fonction du diamètre du grain.

La stabilité du site sous-marin varie ainsi à la fois dans le temps et dans l'espace. Les périodes de calme météorologique induisent des faibles amplitudes de houle et donc des fonds calmes, par contre les tempêtes vont engendrer des transports importants de grains fins peu profonds situés dans des zones très exposées. De tels sédiments pourront également être remaniés par des houles moins fortes qui seront, par contre, insuffisantes pour faire osciller des grains plus gros, plus profonds ou moins exposés.

Pour proposer une interprétation quantitative de phénomènes naturels aussi complexes en raison du caractère aléatoire des tempêtes et donc des remaniements induits il nous a donc fallu réaliser une identification spatio-temporelle de la houle à la suite de campagnes de mesures importantes accompagnées de prélèvements de sédiments in situ.

\subsubsection{Interprétation quantitative}

Interprétation quantitative locale

Dans un premier temps, nous nous intéressons à un site sous-marin donné, non perturbé par des apports de sédiments venus des régions voisines. 


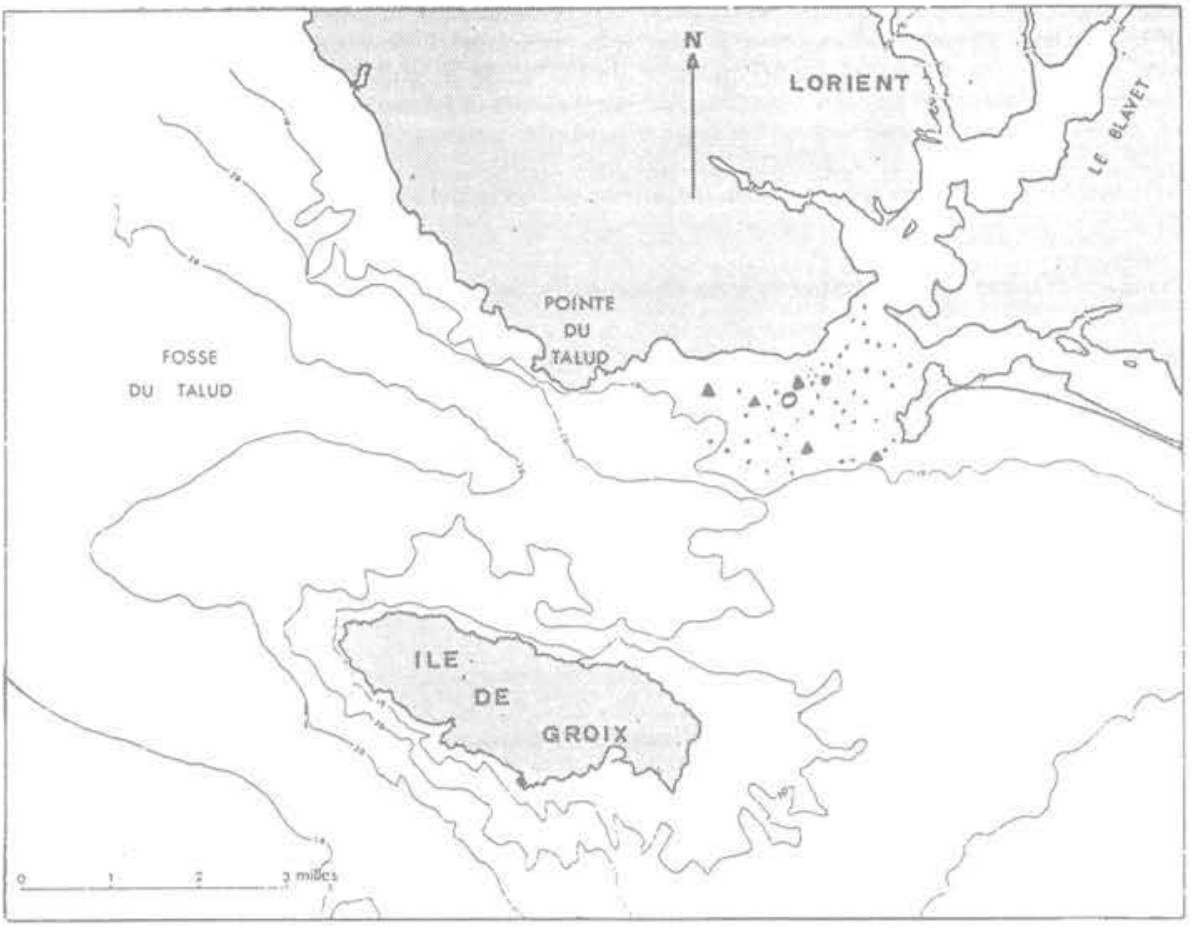

Fig. 1. - Zone étudiée.
O houle
A courants
prélèvements

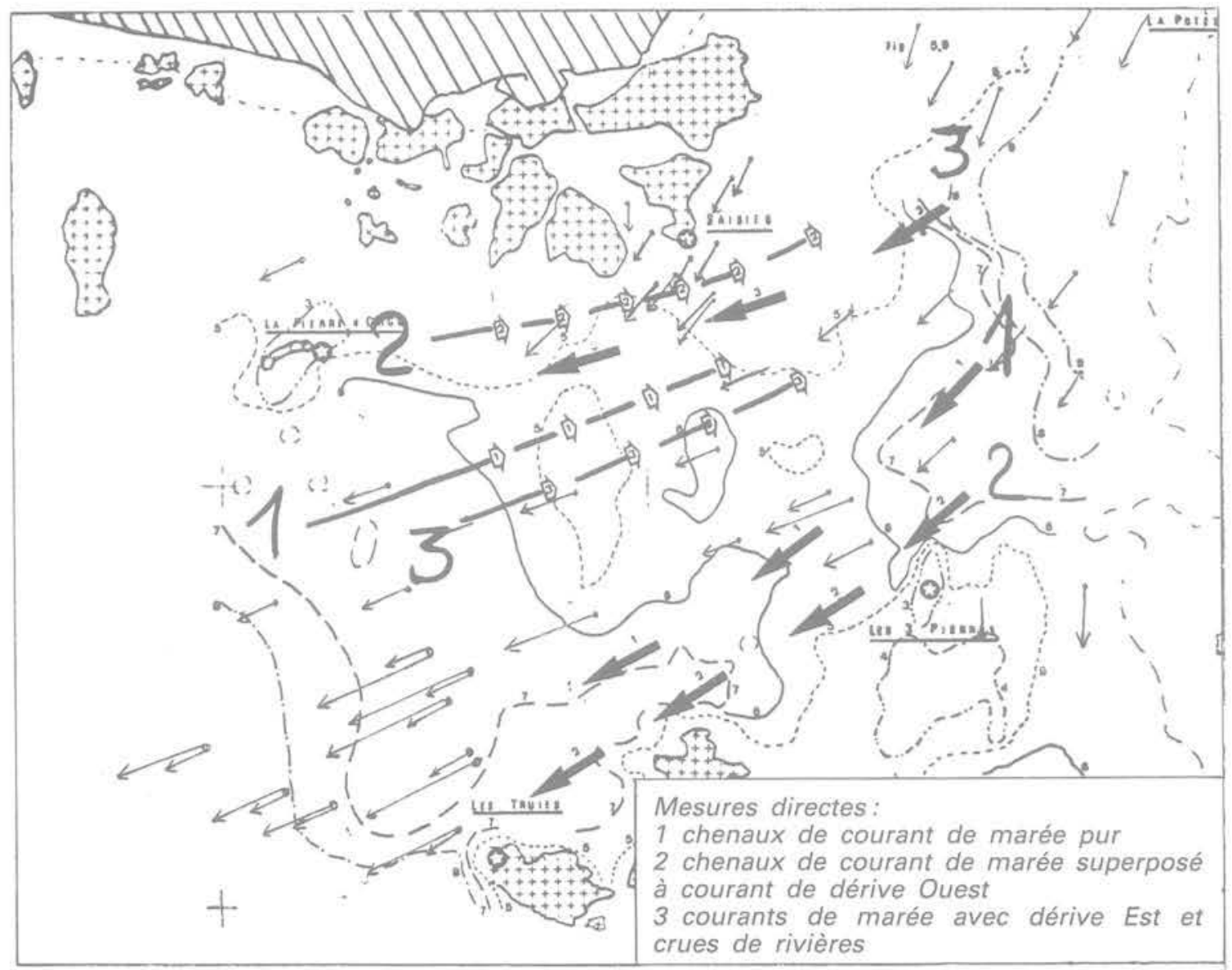

Fig. 2. - Mesures indirectes: les petits vecteurs (la direction est figurative) représentent l'intensité du courant moyen calculé en un point d'après le $D_{\max } d u$ sol sous-marin. Deux vecteurs ont été représentés lorsqu'il y a trois populations. 
On peut considérer que la houle induit sur le fond une vitesse comparable, en première approximation, à celle qu'induirait un courant et appliquer alors la loi de BONNEFILLE vue précédemment, la vitesse maximale sur le fond étant donnée par la relation:

$$
\mathrm{U}=\frac{\pi \mathrm{H}}{\mathrm{Tsh} 2 \pi} \frac{\mathrm{d}}{\mathrm{L}}
$$

$\mathrm{H}$ : amplitude de la houle

$T$ : période

L: longueur d'onde

d: profondeur

Pour connaître la probabilité de remaniement des grains en place localement, il nous faut déterminer la probabilité d'occurrence des houles d'amplitude supérieure ou égale à la houle critique $\mathrm{H}_{\mathrm{c}}$ qui engendre la contrainte critique $\tau_{c}$ de début d'entraînement.

Pour cela, nous avons exploité de longues (près de 7 ans) campagnes de mesures qui nous ont permis de connaître les caractéristiques des vagues et d'en déduire des estimations de la houle décennale, voire centennale appelée aussi houle de projet en raison de son utilisation dans les calculs d'ouvrages maritimes.

\section{Interprétation quantitative régionale}

Sur l'ensemble de la région choisie, une houle donnée a une amplitude très variable en raison de sa variabilité avec la profondeur: c'est ainsi que si $\mathrm{H}_{0}$ est la hauteur large, la hauteur $\mathrm{H}$ en un point de profondeur d sera:

$$
H=\frac{H_{0}}{\operatorname{th} 2 \pi^{d}\left(1+\frac{1}{\operatorname{sh} 4 \pi \frac{d}{L}}\right)}
$$

L'atténuation dépend donc également des obstacles rencontrés antérieurement par les vagues, en conséquence il est indispensable de tracer des «plans de houlex indiquant leur amortissement sur cette région en fonction de la réfraction sur le fond et des angles formés par une direction de propagation donnée avec les lignes bathymétriques.

Pour estimer l'amortissement moyen sur une longue période nous avons pondéré les différents plans de houle, tracés selon les directions d'incidence, en fonction de leur probabilité d'occurrence.

Nous avons ainsi pu obtenir une image moyenne de l'état de la mer sur l'ensemble de la région choisie.

L'analyse granulométrique et sédimentologique des prélèvements en 70 points largement répartis, sur cette zone nous a permis d'en connaître les caractéristiques. L'observation des échantillons à la loupe binoculaire a confirmé que la répartition morphoscopique des grains en nature était très éloignée de celle que lon utilisait pour établir des modèles en laboratoire (population unimodale de billes sphériques).

Nous avons donc réalisé l'interprétation locale du 3.3.1. en ces 70 points et nous avons pu établir une cartographie régionale des sols mobiles fonction de la dimension des grains en place, de la profondeur et de l'amortissement de la houle.

\subsubsection{Synthèse de l'interprétation des phénomènes naturels et de leurs mesures}

La méthodologie que nous venons de décrire peut être parfaitement applicable sur d'autres sites marins dont les sols sont frottants.

D'une façon générale, il sera toujours possible de

\begin{tabular}{|c|c|c|}
\hline $\begin{array}{c}\text { Mesures de } \\
\text { houle en un } \\
\text { point }\end{array}$ & $\begin{array}{l}\text { Dépouille- } \\
\text { ment } \\
\text { et analyse } \\
\text { statique }\end{array}$ & $\begin{array}{c}\text { Image dans } \\
\text { le } \\
\text { temps de la } \\
\text { mer en un } \\
\text { point }\end{array}$ \\
\hline $\begin{array}{l}\text { Plans de ré- } \\
\text { fraction de } \\
\text { la houle dans } \\
\text { la région }\end{array}$ & $\begin{array}{c}\text { Pondération } \\
\text { selon l'oc- } \\
\text { currence des } \\
\text { incidences }\end{array}$ & $\begin{array}{c}\text { Image } \\
\text { moyenne } \\
\text { de l'état de } \\
\text { la mer sur } \\
\text { l'ensemble } \\
\text { de la région }\end{array}$ \\
\hline $\begin{array}{l}\text { Prélèvements } \\
\text { de sols } \\
\text { marins } \\
\text { sur l'en- } \\
\text { semble } \\
\text { de la région }\end{array}$ & $\begin{array}{c}\text { Analyse ex- } \\
\text { périmentale } \\
\text { des sols }\end{array}$ & $\begin{array}{l}\text { Image de la } \\
\text { diversité et } \\
\text { de l'hétéro- } \\
\text { généité des } \\
\text { sols dans la } \\
\text { région }\end{array}$ \\
\hline
\end{tabular}
suivre les schémas de synthèse suivants:

\subsection{Estimations du comportement du sol marin}

Selon l'approche souhaitée, il est possible de proposer différentes estimations du comportement du sol marin.

- La modélisation de la probabilité de remaniement des grains est calculée à partir du début de leur oscillation.

- La modélisation quantitative du remaniement repose sur l'évaluation de la quantité de matériaux en place susceptibles d'être remaniés et fait ainsi apparaître les régions où le sol est mobile.

- La modélisation des remaniements exceptionnels permet de comparer les "sensibilités * des sols aux contraintes engendrées par les tempêtes exceptionnelles et de mettre en évidence la notion de "sol mobilisable».

Nous allons étudier successivement ces trois estimations.

\subsubsection{Modélisation de la probabilité de remanie- ment}

Comme l'a montré C. MIGNIOT (1981) la mise en mouvement des grains apparaît dès que la contrainte tractrice $\tau_{0}$ sur le fond, engendrée par le déplacement des particules de la couche mobile sous l'action de la houle, est supérieure à la contrainte critique de cisaillement $\tau_{c}$ fonction du diamètre des grains. 
Ces contraintes de cisaillement s'expriment selon les relations:

$$
\begin{gathered}
\tau_{0}=1000 \frac{8 \pi \nu}{T^{3}} \frac{H}{s h 2 \pi \frac{d}{L}} \\
\tau_{c}=K \frac{\rho_{s}-\rho_{0}}{\rho_{0}}
\end{gathered}
$$

avec: v:viscosité cinétique de l'eau

T:période de la houle

$\mathrm{H}$ :amplitude

L:longueur d'onde

d:profondeur

$\rho_{\mathrm{s}}$ :masse spécifique du sédiment

$\rho_{0}$ :masse spécifique de l'eau

$\mathrm{D}$ : diamètre

g:accélération de la pesanteur

K:coefficient empirique

Soit $\mathrm{H}_{c}$ la houle critique en un point, c'est-à-dire l'amplitude engendrant la contrainte critique $\tau_{c}$, nous proposons une régionalisation de la probabilité de remaniement par comparaison, sur l'ensemble du site, des occurrences des houles supérieures ou égales aux houles critiques.

Cette régionalisation fait apparaître une probabilité de remaniement des sols frottants faible à l'est et au sud de la région, et plus important au centre et à l'ouest.

\subsubsection{Modélisation quantitative du remaniement}

Pour évaluer la quantité de matériaux remaniés par la houle, nous proposons une interprétation probabiliste du modèle de MEYER-PETER en intégrant l'ensemble des houles engendrant des contraintes supérieures à la contrainte critique en un point donné et en les pondérant par leur probabilité d'occurrence:

$$
\left\{\begin{array}{r}
Q=K \sum_{1=1}^{N}\left(\tau_{0}(\mathrm{l})-\tau_{c}\right)^{1,5} \mathrm{P}(\mathrm{I}) \\
\text { avec } \tau_{0}(\mathrm{I})>\tau_{c}
\end{array}\right.
$$

P(I) étant la probabilité d'occurrence de la houle d'amplitude I.

Nous avons ainsi pu faire apparaître une régionalisation du sol mobile statistique (fig. 5) qui est assez proche de résultats obtenus grâce à la modélisation précédente.

Il est à noter que nous avons obtenu une cartographie très proche en appliquant un modèle obtenu sur un autre site naturel à partir de taux de comptage d'indicateurs radioactifs.

\subsubsection{Modélisation des remaniements exceptionnels}

Nous proposons ici, une nouvelle estimation reposant sur l'évaluation, en un point quelconque de la région, du diamètre $D_{\text {an }}$ du plus gros grain remanié par la plus forte tempête (d'amplitude $\mathrm{H}_{a n}$ ) de l'année.

$$
\mathrm{D}_{\mathrm{an}}=\frac{\pi \mathrm{H}_{\mathrm{an}}}{500 \mathrm{~T}_{\mathrm{an}}^{2} \operatorname{sh} 2 \pi \frac{\mathrm{d}}{\mathrm{L}_{\mathrm{an}}}}
$$

la longueur d'onde $L_{\text {an }}$ étant calculée par résolution de l'équation implicite

$$
-\mathrm{L}_{\mathrm{an}}=\frac{\mathrm{gT}_{\mathrm{an}}^{2}}{2 \pi} \text { th2 } 2 \pi \frac{\mathrm{d}}{\mathrm{L}_{\mathrm{an}}}
$$

d: profondeur

$\mathrm{L}_{\mathrm{an}}$ : longueur d'onde

Ce modèle est donc calculé à partir de la houle seule, il permet de connaître le comportement « virtuel * du sol marin. En effet, la régionalisation du paramètre $D_{\text {an }}$ fait apparaître deux zones nettement différenciées:

- la zone S dont les sols marins ne seront remaniés exceptionnellement que s'ils sont composés de grains fins;

- la zone D sur laquelle les tempêtes remanieront également des grains beaucoup plus grossiers.

On constate, bien que les délimitations ne soient pas identiques aux précédentes, que les zones est et sud du site étudié semblent stables, la zone nord-ouest l'étant beaucoup moins, confirmant ainsi les indications obtenues avec les autres modèles.

\subsection{Identification statistique des paramètres caractérisant la tenue des sols marins}

\subsubsection{Processus naturel et modèle de laboratoire}

En un point de prélèvement, l'histoire du sédiment en place peut être diverse et complexe, en effet une tempête a pu «marquer» le sol selon des processus très différents, par exemple:

- mise en suspension de l'ensemble du sédiment en place précédemment et remplacement par une nouvelle population dont la dimension des plus gros grains sera caractéristique de l'importance de la perturbation;

- mise en suspension de la partie la plus fine seulement:

- mise en suspension partielle combinée avec un apport de sédiments en provenance d'une autre zone;

- absence d'oscillation de la population en place, mais apport supplémentaire de sêdiments plus fins extérieurs;

- absence totale de modification du sol sous-marin.

La complexité de la superposition, sur l'ensemble du site, de phénomènes aussi divers est encore accrue par la possibilité de génération d'une nouvelle famille de grains par altération de la roche sous-jacente.

Nous avons cherché, dans les paragraphes précédents à utiliser des modèles obtenus dans des conditions idéales de laboratoire (écoulement laminaire, population unimodale de billes sphériques). Nous avons essayé d'affiner ces modélisations contribuant à améliorer la simulation des phénomènes naturels, ce qui nous a permis d'obtenir différentes estimations de tenue des sols marins assez comparables, en utilisant des modèles reposant sur des approches très variées.

Pour compléter encore ces estimations, et devant l'impossibilité de restituer avec précision l'ahistoire» très tourmentée d'un sol prélevé, nous avons cherché 


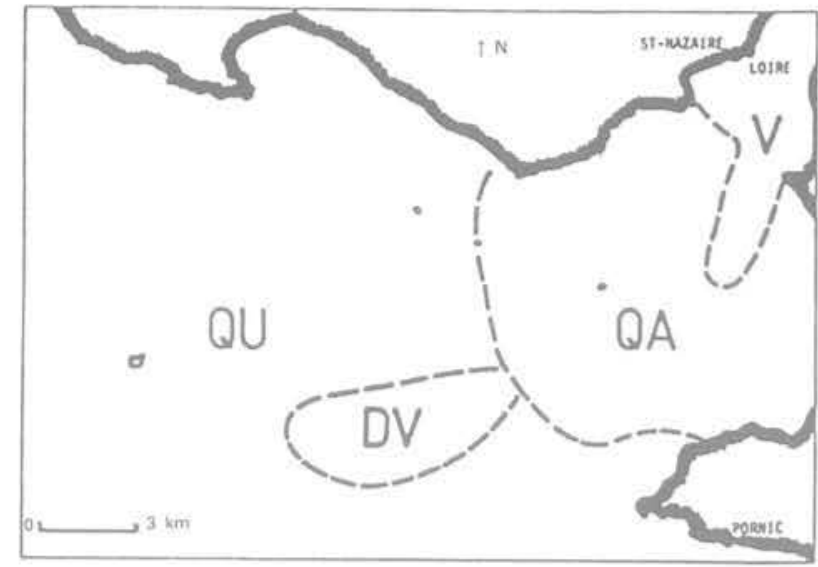

Fig. 3. - Régionalisation des sols en place selon leur nature.

Zone QA: prédominance de quartz anguleux

Zone QU: prédominance de quartz usés et de coquilles

Zone $\mathrm{V}$ : sols cohésifs (apports de Loire)

Zone DV: dépôt de dragage (apports cohésifs artificiels)

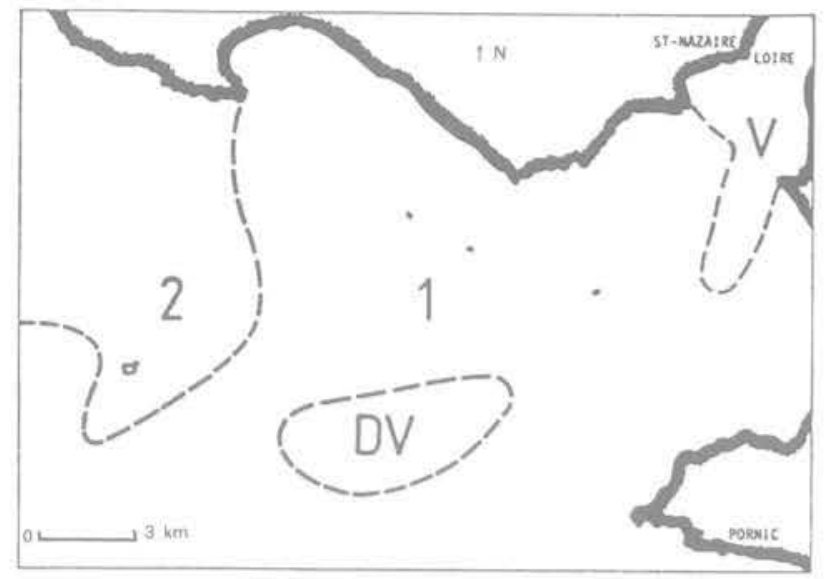

Fig. 5. - Régionalisation du sol mobile.

Zone 1 : débit annuel de matériaux frottants inférieurs à $16 \mathrm{~m}^{3} / \mathrm{m} /$ an

Zone 2: débit supérieur à $16 \mathrm{~m}^{3} / \mathrm{m} / \mathrm{an}$

Zone V: sols cohésifs (apports de Loire)

Zone DV: dépôt de dragage (apports cohésifs artificiels)

à tester la fiabilité de certains paramètres considérés comme des images du sol marin.

\subsubsection{Etude corrêlative}

L'identification statistique des paramètres caractérisant la tenue des sols marins repose sur l'étude des liaisons fonctionnelles éventuelles entre les différents paramètres locaux, images de la répartition de la houle et du sol marin sur l'ensemble de la région.

La variabilité de l'amplitude de la houle est caractérisée par la distribution spatiale de son amortissement moyen a.

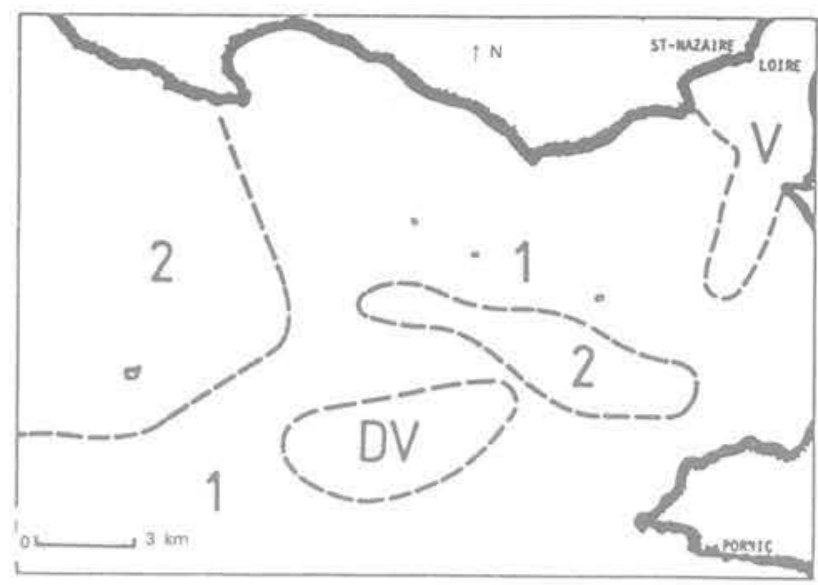

Fig. 4. - Régionalisation de la probabilité de remaniement.

Zone 1: nombre annuel de jours de remaniement inférieurs à 16

Zone 2: nombre annuel de jours de remaniement supérieurs à 16

Zone V: sols cohésifs (apports de Loire)

Zone DV: dépôt de dragage (apports cohésifs artificiels)

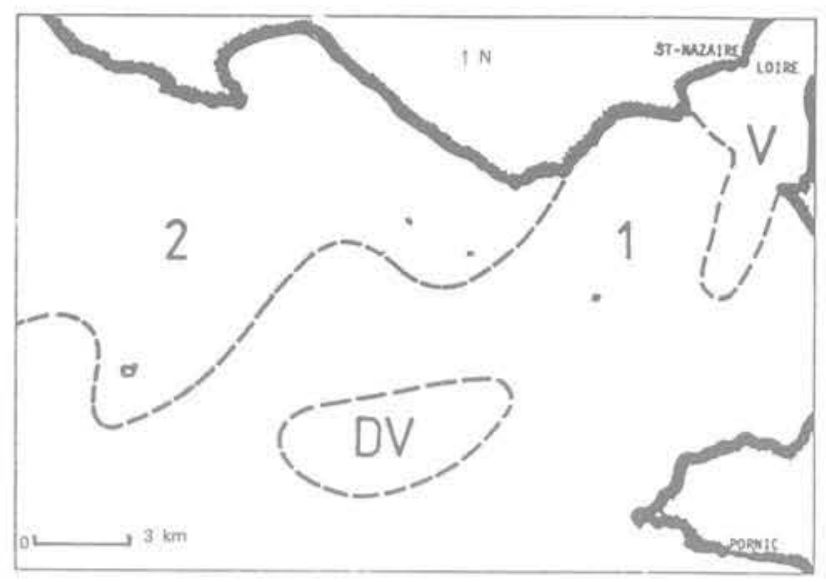

Fig. 6. - Régionalisation du sol mobilisable

Zone 1: diamètre du plus gros grain de quartz remanié annuellement inférieur à $1 \mathrm{~mm}$

Zone 2: diamètre du plus gros grain de quartz remanié annuellement supérieur à $1 \mathrm{~mm}$

Zone V: sols cohésifs (apports de Loire)

Zone DV: dépôt de dragage (apports cohésifs artificiels)

La diversité du sol marin peut être caractérisée par différents paramètres:

- diamètres $\mathrm{D}_{50}, \overline{\mathrm{D}}, \mathrm{D}_{\max }$;

- fraction granulométrique prépondérante:

- proportion de grains usés: u (obtenue par observation et comptage à la loupe binoculaire).

La validité de l'hypothèse de corrélation a été vérifiée par des tests qui ont donné des résultats identiques:

- Absence de liaison fonctionnelle entre la houle (a) et les paramètres $D_{50}, \bar{D}, \bar{G}$. 
- Validité de la liaison fonctionnelle entre la houle (a) et le diamètre maximum $\left(D_{\max }\right)$ d'une part et le degré d'usure des grains $(u)$ d'autre part.

S'il paraît tout à fait naturel que le degré d'usure soit lié à la houle, en raison du transport des grains par saltation que celle-ci engendre, l'autre corrélation (entre a et $\mathrm{D}_{\text {max }}$ ) montre l'intérêt de la prise en compte, dans une modélisation, du paramètre $\mathrm{D}_{\max }$ (diamètre maximum d'une famille de grains) dont l'importance avait déjà été mis en évidence précédemment lors de l'étude de l'influence des courants sur la tenue des sols marins.

\section{CONCLUSIONS - L'INTERPRÉTATION GÉOTECHNIQUE}

La méthode qui consiste à appliquer en nature les lois tirées des essais de laboratoire d'hydraulique s'avère donc fructueuse à condition:

- d'utiliser des lois de synthèse valables pour toute densité relative et toute viscosité

- de les appliquer à un paramètre dimensionnel très particulier, le $\mathrm{D}_{\max }$, borne d'une population statistique.

Dans un sol frottant de granulométrie étalée, sous l'effet des forces tractrices des courants ou de la houle sur le fond, il y aura donc érosion puis dépôt d'une fraction de la population des grains, et le diamètre du plus gros grain remué; $D_{\max }$, correspondra à la force tractrice maximale sur le fond, liée à la vitesse orbitale des houles ou la vitesse moyenne des courants.

Cette action des courants et des houles surtout, dont la répartition dans l'espace est variée, a permis par comparaison entre modélisations statistiques de mettre en lumière la très bonne corrélation entre la hauteur des houles maximales $\mathrm{H}_{\max }$; en un point et le $\mathrm{D}_{\max }$ du sol sous-marin, ceci pour des périodes inférieures ou égales à un an.

Il n'en est pas de même du diamètre moyen, pourtant couramment utilisé, et qui par conséquent n'a aucune signification dynamique.

En outre la corrélation $\mathrm{H}_{\max }$ /degré d'usure des grains, fait prévoir une prédominance des déplacements alternatifs sur les déplacements vrais.

Les phénomènes de surface reçoivent donc là des explications satisfaisantes et l'on constate une bonne adéquation entre les modèles de transport intégrant les paramètres précédents et les données d'observation ou les résultats d'autres modèles.
Tout ceci est intégré dans un temps court, plurimensuel. Or, l'interprétation géotechnique conduit à faire intervenir la profondeur au sein du sol, donc l'intégration de ces phénomènes dans un temps plus long, pluri-annuel

Théoriquement, si les conditions décennales ou centenales sont très marquées, et océanographiquement bien connues, il peut paraître facile de distinguer sur une carotte les différentes zones par la variation des $\mathrm{D}_{\max }$; dans certains cas cette détermination est effectivement concluante pour des sables faciles à prélever au Kullenberg, de faible compacité et donc récemment déposés.

Ainsi, très logiquement, la continuité du processus sédimentaire en un point, apparaît une condition nécessaire à l'application de règles simples.

A l'inverse, les arrêts de sédimentation, les érosions, se traduisant par des fonds durs, des effets de voute intergranulaire, mettent en lumière l'importance qu'aurait pour la solution de problèmes d'affouillement par exemple, la connaissance de la signification sédimentologique d'un paramètre géotechnique aussi simple, mais aussi difficile à mesurer, que l'indice des vides en place.

\section{BIBLIOGRAPHIE}

BONNEFILLE, R. (1976). - Hydraulique Maritime. Ed. Masson.

CHAPON, J. (1978). - Travaux Maritimes. - Ed. Eyrolles.

GROVEL, A. (1979), - Essai de corrélation entre les lois de début d'entraînement des sédiments sous l'action de la houle et des courants et les analyses granulométriques de sables. - Journal de Recherche Océanographique, vol. IV, bulletin $n^{\circ}$, 1979.

LE TIRANT, P. (1976). - Reconnaissance des sols en mers. - Ed. Technip.

MIGNIOT, C. (1981). - Erosion et sédimentation en mer et en rivière; in la Pratique des Sols et des Fondations. - Ed. Moniteur des Travaux Publics.

SILVESTER, R. (1974). - Coastal Engineering. Developments in geotechnical engineering. - Ed. Elsevier.

THOMAS, P. (1981). - Contribution à l'étude en nature des houles et de leur influence sur la tenue des sols frottants dans une zone littorale estuarienne. - Thèse Docteur-Ingénieur Nantes. 
